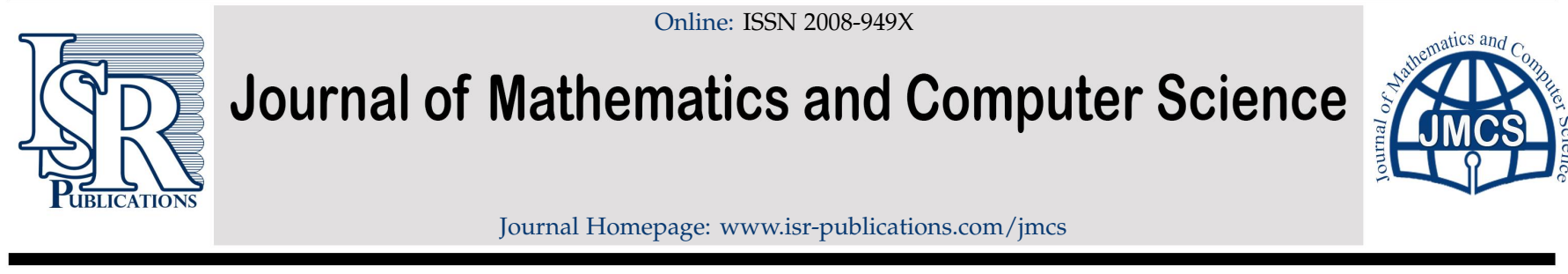

\title{
Asymptotic behavior of traveling waves for non-quasi- monotone system with delay
}

Yong-Hui Zhou*, Wen-Di Li, Yan-Ru Che

School of Mathematics and Statistics, HeXi University, Zhangye, Gansu 734000, P. R. China.

\begin{abstract}
This paper is concerned with a population dynamic model with delay. In this work, by rewriting the equation and using the Ikehara's theorem, we show the exact asymptotic behavior of the profile as $\xi \rightarrow-\infty$ for critical speed.
\end{abstract}

Keywords: Traveling waves, Ikehara's theorem, asymptotic behavior.

2020 MSC: 34K18, 37G10, 37G05

(C)2022 All rights reserved.

\section{Introduction}

In this work, we are concerned with the asymptotic behavior of non-monotone traveling waves of the delayed reaction diffusion equation without quasi-monotonicity

$$
\left\{\begin{array}{l}
u_{1 t}(t, x)=d u_{1 x x}(t, x)-a_{11} u_{1}(t, x)+a_{12} u_{2}(t, x), \\
u_{2 t}(t, x)=-a_{22} u_{2}(t, x)+\widetilde{g}\left(u_{1}(t-\tau, x)\right),
\end{array}\right.
$$

where $d, a_{11}, a_{12}$, and $a_{22}$ are positive constants, $u_{1}(t, x)$ and $u_{2}(t, x)$ denote the spatial densities of infectious agents and the infective human population at time $t \geqslant 0$, respectively. $1 / a_{11}$ is the mean lifetime of the agents in the environment, $1 / a_{22}$ is the mean infectious period of the infective human, $a_{12}$ is the multiplicative factor of the infectious agents due to the human population, $\widetilde{g}\left(u_{1}\right)$ is the force of infection on human population due to a concentration $u_{1}$ of the infectious agents and $\tau$ is the disease latent period.

Over the years, there have been many important monotone results $[9,11,15,16]$ on traveling wave solutions for reaction-diffusion scalar equations and systems.

However, on the traveling wave solutions of reaction-diffusion equations and systems without quasimonotonicity, it seems that few results $[2,3,6,8]$. Wu and $\mathrm{Li}[10,12]$ established the existence of traveling wave solutions for some complicated non-local reaction-diffusion equations with delay and non-local diffusion term equations with delay the idea of auxiliary equations and Schauders fixed point theorem

\footnotetext{
*Corresponding author

Email addresses: 2823877618@qq. com (Yong-Hui Zhou), 1wd12280213@163.com (Wen-Di Li), 2496231775@qq.com (Yan-Ru Che)
} doi: $10.22436 /$ jmcs.025.01.03

Received: 2020-12-30 Revised: 2021-03-30 Accepted: 2021-03-31 
$[7,8]$, respectively. In particular, $\mathrm{Wu}$ [12] applied the method to solve the existence of traveling wave solutions of a class of non-monotone integral equations. As an application of this result, the existence of traveling wave solutions of the following epidemic system with distributed delay

$$
\left\{\begin{array}{l}
u_{1 t}(t, x)=u_{1 x x}(t, x)-u_{1}(t, x)+u_{2}(t, x), \\
u_{2 t}(t, x)=-\beta u_{2}(t, x)+\int_{0}^{\infty} g\left(u_{1}(t-s, x)\right) P(d s),
\end{array}\right.
$$

has been obtained. It is easy to see that if $\mathrm{P}(\cdot)$ is Dirac function $\delta(\cdot)$ and (1.2) is the system (1.1). To the best of our knowledge, the asymptotic behavior of non-monotone traveling waves of such equations remain open and seem to be very interesting and challenging problems. The purpose of this work is to establish the asymptotic behavior of the non-monotone traveling waves of (1.1) in the non-monotone monostable case.

\section{Preliminaries and main results}

Notations. Throughout this paper, $C>0$ denotes a generic constant, $C_{i}>0(i=1,2, \ldots)$ represents a specific constant. Let I be an interval. $\mathrm{L}^{2}(\mathrm{I})$ is the space of the square integrable functions defined on $\mathrm{I}$, and $H^{k}(I)(k \geqslant 0)$ is the Sobolev space of the $L^{2}$-function $h(x)$ defined on the interval I whose derivatives $\frac{d^{i}}{d x^{i}} h(i=1,2, \ldots, k)$ also belong to $L^{2}(I) . L_{w}^{2}(I)$ denotes the weighted $L^{2}$-space with a weight function $w(x)>0$ and its norm is defined by $\|h\|_{L_{w}^{2}}=\left(\int_{I} w(x)|h(x)|^{2} d x\right)^{\frac{1}{2}}$, and $H_{w}^{k}(I)$ is the weighted Sobolev space with the norm given by

$$
\|h\|_{H_{w}^{k}}=\left(\sum_{i=0}^{k} \int_{I} w(x)\left|\frac{d^{i}}{d x^{i}} h(x)\right|^{2} d x\right)^{\frac{1}{2}} .
$$

Let $T>0$ be a number and $\mathcal{B}$ be a Banach space. $C([0, T] ; \mathcal{B})$ is the space of $\mathcal{B}$-valued continuous functions on $[0, T] . L^{2}([0, T] ; \mathcal{B})$ is the space of $\mathcal{B}$-valued $L^{2}$-functions on $[0, T]$. The corresponding spaces of $\mathcal{B}$-valued functions on $[0, \infty)$ are defined similarly.

For convenience, we rewrite (1.1) to the following system

$$
\left\{\begin{array}{l}
u_{1 t}(t, x)=u_{1 x x}(t, x)-u_{1}(t, x)+u_{2}(t, x), \\
u_{2 t}(t, x)=-\beta u_{2}(t, x)+g\left(u_{1}(t-\tau, x)\right),
\end{array}\right.
$$

where $\beta=\frac{a_{11}}{a_{22}}, g=\frac{a_{12}}{a_{22}^{2}} \widetilde{g}$.

A traveling wave of Eq. (2.1) connecting $u_{-}$and $u_{+}$is a solution $u(t, x)=\phi(x+c t)=\phi(\xi), \xi=x+c t$, satisfying the following ordinary differential equation

$$
\left\{\begin{array}{l}
c \phi_{1}^{\prime}(\xi)=\phi_{1}^{\prime \prime}(\xi)-\phi_{1}(\xi)+\phi_{2}(\xi) \\
c \phi_{2}^{\prime}(\xi)=-\beta \phi_{2}(\xi)+g\left(\phi_{1}(\xi-c \tau)\right) \\
\phi_{1}( \pm \infty)=u_{1 \pm}, \quad \phi_{2}( \pm \infty)=u_{2 \pm} .
\end{array}\right.
$$

The characteristic equation associated with the linearized equation of $(1.2)$ at $(0,0)$ is

$$
P(\lambda)=\left(\lambda^{2}-c \lambda-1\right)(-c \lambda-\beta)-g^{\prime}(0) .
$$

In addition, throughout this paper, we assume that $\beta>1$ and (1.1) satisfies the initial conditions

$$
\begin{cases}u_{1}(s, x)=u_{10}(s, x), & (s, x) \in[-\tau, \mathbb{R}], \\ u_{2}(0, x)=u_{20}(x), & x \in \mathbb{R} .\end{cases}
$$

We also need the following assumptions for the sake of proving the existence of traveling wave solutions (see [13]): 
$\left(\mathrm{A}_{1}\right) g(0)=\beta K-g(K)=0$ for some $K>0, g^{\prime}(0)>\beta$, and there exists a $v \in(0,1]$ such that $\lim \sup _{u \rightarrow 0^{+}}\left[g^{\prime}(0)-\frac{g(u)}{u}\right] u^{-v}<+\infty ;$

$\left(\mathrm{A}_{2}\right) \min \left\{\mathrm{k}^{*} \mathrm{u}, \mathrm{K}^{+}\right\} \geqslant \frac{1}{\beta} \mathrm{g}(\mathrm{u})>0$ for all $\mathrm{u} \in\left(0, \mathrm{~K}^{+}\right]$and $\mathrm{g}$ is Lipschitz continuous on $\left[0, \mathrm{~K}^{+}\right]$for some $\mathrm{K}^{+} \geqslant \mathrm{K}$, where $\mathrm{k}^{*}=\frac{\mathrm{g}^{\prime}(0)}{\beta}$;

$\left(\mathrm{A}_{3}\right)$ one of the following assumptions holds:

(i) $K \geqslant \frac{1}{\beta} g(u)>u$ for all $u \in(0, K)$;

(ii) $u<\frac{1}{\beta} g(u)<2 K-u$ for $u \in\left[K^{-}, K\right)$ and $u>\frac{1}{\beta} g(u)>2 K-u$ for $u \in\left(K, K^{+}\right]$, where $\mathrm{K}^{-}=\mathrm{k}^{*} \inf _{\mathrm{\eta} \in\left(0, \mathrm{~K}^{+}\right]}\left\{\mathrm{g}(\eta): \frac{1}{\beta} \mathrm{g}(\eta) \leqslant \eta\right\} ;$

(iii) $u<\frac{1}{\beta} g(u)$ for $u \in\left[K^{-}, K\right), u>\frac{1}{\beta} g(u)$ for $u \in\left(K, K^{+}\right]$and there is no pair $0<\gamma_{1}<K<\gamma_{2} \leqslant$ $\mathrm{K}^{+}$such that $\gamma_{1}=\frac{1}{\beta} \mathrm{g}\left(\gamma_{2}\right)$ and $\gamma_{2}=\frac{1}{\beta} \mathrm{g}\left(\gamma_{1}\right)$;

$\left(\mathrm{A}_{4}\right) \mathrm{g}^{\prime \prime}(\mathrm{u})<0$ for all $\mathrm{u} \in(0, \infty)$.

Proposition 2.1 (Existence of traveling waves). Assume that $\left(\mathrm{A}_{1}\right)-\left(\mathrm{A}_{2}\right)$ holds. Then, there exists $\mathrm{c}_{*}>0$ such that

(i) for any $\mathrm{c}>\mathrm{c}_{* \prime},(1.2)$ admits a traveling wave solution $\Phi(\xi):=\left(\phi_{1}(\xi), \phi_{2}(\xi)\right)$ satisfying $\phi_{i}(\xi)=\mathrm{O}\left(\mathrm{e}^{\wedge_{1}(\mathrm{c}) \xi}\right)$ as $\xi \rightarrow-\infty, i=1,2, \phi_{1}(\xi) \in \mathrm{C}\left(\mathbb{R},\left[0, \mathrm{~K}^{+}\right]\right)$and

$$
\begin{aligned}
0<\mathrm{K}^{-} & \leqslant \lim _{\xi \rightarrow+\infty} \inf \phi_{1}(\xi) \leqslant \lim _{\xi \rightarrow+\infty} \sup \phi_{1}(\xi) \leqslant \mathrm{K}^{+}, \\
0 & <\lim _{\xi \rightarrow+\infty} \inf \phi_{2}(\xi) \leqslant \lim _{\xi \rightarrow+\infty} \sup \phi_{2}(\xi) \leqslant \mathrm{K}^{+},
\end{aligned}
$$

moreover, if $\left(A_{3}\right)$ holds, then $\Phi(+\infty)=\left(K, K_{0}\right)$, where $K_{0}=g(K) / \beta$ and $\Lambda_{1}(c)$ is the smallest solution such that the linearized characteristic equation at $(0,0)$ of $(1.2)$ has solutions;

(ii) for $\mathrm{c}=\mathrm{c}_{*},(1.2)$ admits a traveling wave solution with the wave speed $\mathrm{c}_{*}$;

(iii) for all $\mathrm{c} \in\left(0, \mathrm{c}_{*}\right),(1.2)$ admits no such wave solution with the wave speed $\mathrm{c}$.

Lemma $2.2([4])$. Let $\mathrm{F}(\lambda):=\int_{-\infty}^{0} \phi(\xi) e^{-\lambda \xi} \mathrm{d} \xi$, where $\phi(\xi)$ is a positive increasing function for $\xi \in \mathbb{R}$. Suppose that $F(\lambda)$ can be written in the form

$$
F(\lambda)=\frac{H(\lambda)}{(\alpha-\lambda)^{k+1}}
$$

where $\mathrm{k}>-1$ and $\mathrm{H}(\lambda)$ is analytic in the strip $0<\operatorname{Re} \lambda \leqslant \alpha$ for some $\alpha>0$, then

$$
\lim _{\xi \rightarrow-\infty} \frac{\phi(\xi)}{|\xi|^{k} e^{\lambda \xi}}=\frac{H(\lambda)}{\Gamma(\lambda+1)}
$$

Next, we state our main result about the asymptotic behavior of traveling wave solutions of (1.1).

Theorem 2.3. Assume that $\left(\mathrm{A}_{1}\right)-\left(\mathrm{A}_{4}\right)$ hold. Let $\left(\phi_{1}, \phi_{2}\right)$ be the solution of $(2.2)$, then there exist constants $\theta_{i}=\theta_{i}\left(\phi_{1}, \phi_{2}\right), i=1,2$, such that

$$
\lim _{\xi \rightarrow-\infty} \frac{\phi_{i}\left(\xi+\theta_{i}\right)}{|\xi|^{k} e^{\lambda_{1} \xi}}=1
$$

where $\mathrm{k}=0$ for $\mathrm{c}>\mathrm{c}^{*}$ and $\mathrm{k}=1$ for $\mathrm{c}=\mathrm{c}^{*}$.

Proof. By Proposition 2.1, it is easy to obtain that, for any $0<k<\lambda_{1}, \phi_{i}(\xi)=O\left(e^{k \xi}\right)$ as $\xi \rightarrow-\infty, i=1,2$, where $\lambda_{1}$ is the smallest positive real root of $\mathrm{P}(\lambda)=0$ for (2.1). Let denote's

$$
\Phi_{i}(\lambda):=\int_{\mathbb{R}} \phi_{i}(\xi) e^{-\lambda \xi} d \xi, \quad i=1,2
$$


Then we get that $\Phi_{i}(\lambda)<\infty$ for all complex $\lambda$ with $\operatorname{Re} \lambda \in\left(0, \lambda_{1}\right)$. By integration by parts, we have

$$
\int_{\mathbb{R}} \phi_{i}^{\prime}(\xi) e^{-\lambda \xi} d \xi=\lambda \Phi_{i}(\lambda), i=1,2, \operatorname{Re} \lambda \in\left(0, \lambda_{1}\right),
$$

and

$$
\int_{\mathbb{R}} \phi_{i}^{\prime \prime}(\xi) e^{-\lambda \xi} d \xi=\lambda^{2} \Phi_{i}(\lambda), \quad i=1,2, \quad \operatorname{Re} \lambda \in\left(0, \lambda_{1}\right) .
$$

Equations (2.2) can be rewritten in the following integral form

$$
\begin{aligned}
& \phi_{1}(\xi)=\frac{1}{\lambda_{1}^{+}-\lambda_{1}^{-}}\left[\int_{-\infty}^{\xi} e^{\lambda_{1}^{-}(\xi-s)} \phi_{2}(s) d s+\int_{\xi}^{+\infty} e^{\lambda_{1}^{+}(\xi-s)} \phi_{2}(s) d s\right], \\
& \phi_{2}(\xi)=\frac{1}{c} \int_{-\infty}^{\xi} e^{-\frac{\beta}{c}(\xi-s)} g\left(\phi_{1}(s-c \tau)\right) d s,
\end{aligned}
$$

where $\lambda_{1}^{-}=\frac{\mathfrak{c}-\sqrt{c^{2}+4}}{2}<0$ and $\lambda_{1}^{+}=\frac{\mathfrak{c}+\sqrt{c^{2}+4}}{2}>0$ are the solutions of $\lambda^{2}-c \lambda-1=0$. Moreover, the integral form (2.3) and (2.4) imply that

$$
\begin{aligned}
& \phi_{1}^{\prime}(\xi)=\frac{1}{\lambda_{1}^{+}-\lambda_{1}^{-}}\left[\lambda_{1}^{-} \int_{-\infty}^{\xi} e^{\lambda_{1}^{-}(\xi-s)} \phi_{2}(s) d s+\lambda_{1}^{+} \int_{\xi}^{+\infty} e^{\lambda_{1}^{+}(\xi-s)} \phi_{2}(s) d s\right], \\
& \phi_{2}^{\prime}(\xi)=-\frac{\beta}{c^{2}} \int_{-\infty}^{\xi} e^{-\frac{\beta}{c}(\xi-s)} g\left(\phi_{1}(s-c \tau)\right) d s .
\end{aligned}
$$

Let $0<\mathrm{k}<\lambda_{1}$, since $\phi_{2}(\xi)=\mathrm{O}\left(e^{k \xi}\right)$ as $\xi \rightarrow-\infty$, there exists a $\xi_{1}<0$ such that $\left|\phi_{2}(\xi)\right| \leqslant M e^{k \xi}$ for $\xi \leqslant \xi_{1}$, we obtain

$$
\int_{-\infty}^{\xi}\left|e^{\lambda_{1}^{-}(\xi-s)} \phi_{2}(s)\right| d s \leqslant M \int_{-\infty}^{\xi} e^{\lambda_{1}^{-}(\xi-s)} e^{k s} d s=\frac{M e^{k \xi}}{k-\lambda_{1}^{-}}
$$

and

$$
\begin{aligned}
\int_{\xi}^{+\infty}\left|e^{\lambda_{1}^{+}(\xi-s)} \phi_{2}(s)\right| d s & =\int_{\xi}^{\xi_{1}}\left|e^{\lambda_{1}^{+}(\xi-s)} \phi_{2}(s)\right| d s+\int_{\xi_{1}}^{+\infty}\left|e^{\lambda_{1}^{+}(\xi-s)} \phi_{2}(s)\right| d s \\
& \leqslant M \int_{\xi}^{\xi_{1}} e^{\lambda_{1}^{+}(\xi-s)} e^{k s} d s+e^{\lambda_{1}^{+} \xi} \int_{\xi_{1}}^{+\infty} e^{-\lambda_{1}^{+} s}\left|\phi_{2}(s)\right| d s \\
& \leqslant \frac{M e^{k \xi}}{\lambda_{1}^{+}-k}+M_{1} e^{\lambda_{1}^{+} \xi}
\end{aligned}
$$

where $M_{1}=\int_{\xi_{1}}^{+\infty} e^{-\lambda_{1}^{+} s}\left|\phi_{2}(s)\right| d s<\infty$. Thus, it follows that $\phi_{1}^{\prime}(\xi)=\mathrm{O}\left(e^{k \xi}\right)$, and similarly $\phi_{2}^{\prime}(\xi)=$ $\mathrm{O}\left(\mathrm{e}^{\mathrm{k} \xi}\right)$ as $\xi \rightarrow-\infty$. Since $\mathrm{g} \in \mathrm{C}^{2}$, we get

$$
\mathrm{g}\left(\phi_{1}(\xi-c \tau)\right)-\mathrm{g}^{\prime}(0) \phi_{1}(\xi-c \tau)=\mathrm{O}\left(\left|\phi_{1}(\xi-c \tau)\right|^{2}\right)=\mathrm{O}\left(\left|\phi_{1}(\xi)\right|^{2}\right)=\mathrm{O}\left(e^{2 k \xi}\right)
$$

as $\xi \rightarrow-\infty$ for any $0<k<\lambda$. Now we define the functions $Q_{i}(\lambda), i=1,2$,

$$
\mathrm{Q}_{1}(\lambda):=\int_{\mathbb{R}} \phi_{2}(\xi) e^{-\lambda \xi} \mathrm{d} \xi, \quad \mathrm{Q}_{2}(\lambda):=\int_{\mathbb{R}}\left[g^{\prime}(0) \phi_{1}(\xi-c \tau)-g\left(\phi_{1}(\xi-c \tau)\right)\right] e^{-\lambda \xi} \mathrm{d} \xi,
$$

are analytic in the strip $\operatorname{Re} \lambda \in\left(0,2 \lambda_{1}\right)$. At the same time, it is easy to see that $Q_{\mathfrak{i}}(\lambda)>0, i=1,2, \operatorname{Re} \lambda \in$ $\left(0,2 \lambda_{1}\right)$. Therefore, $(2.2)$ can be rewritten in the following form

$$
\begin{aligned}
\phi_{1}^{\prime \prime}(\xi)-c \phi_{1}^{\prime}(\xi)-\phi_{1}(\xi) & =-\phi_{2}(\xi), \\
-c \phi_{2}^{\prime}(\xi)-\beta \phi_{2}(\xi)+g^{\prime}(0) \phi_{1}(\xi-c \tau) & =g^{\prime}(0) \phi_{1}(\xi-c \tau)-g\left(\phi_{1}(\xi-c \tau)\right) .
\end{aligned}
$$


Multiplying (2.5) and (2.6) by $e^{-\lambda \xi}$ and integrating both sides of the equations from $-\infty$ to $+\infty$, we get

$$
\begin{aligned}
\left(\lambda^{2}-c \lambda-1\right) \Phi_{1}(\lambda) & =-Q_{1}(\lambda), \\
(-c \lambda-\beta) \Phi_{2}(\lambda)+g^{\prime}(0) e^{-\lambda c \tau} \Phi_{1}(\lambda) & =Q_{2}(\lambda) .
\end{aligned}
$$

Thus,

$$
\begin{aligned}
\Phi_{1}(\lambda) & =-\frac{\mathrm{Q}_{1}(\lambda)}{\lambda^{2}-c \lambda-1}, \quad \operatorname{Re} \lambda \in\left(0, \lambda_{1}\right), \\
P(\lambda) \Phi_{2}(\lambda) & =\left(\lambda^{2}-c \lambda-1\right) Q_{2}(\lambda)+g^{\prime}(0)\left[e^{-\lambda c \tau}-1\right] Q_{1}(\lambda), \quad \operatorname{Re} \lambda \in\left(0, \lambda_{1}\right) .
\end{aligned}
$$

Denoting $F(\lambda):=\int_{-\infty}^{0} \phi_{2}(\xi) e^{-\lambda \xi} d \xi$, by (2.7), $F(\lambda)$ can be rewritten as

$$
F(\lambda)=\frac{\left(\lambda^{2}-c \lambda-1\right) Q_{2}(\lambda)+g^{\prime}(0)\left[e^{-\lambda c \tau}-1\right] Q_{1}(\lambda)}{P(\lambda)}-\int_{0}^{+\infty} \phi_{2}(\xi) e^{-\lambda \xi} d \xi:=\frac{H(\lambda)}{\left(\lambda_{1}-\lambda\right)^{k+1}},
$$

where $k=0$ for $c>c_{*}$ and $k=1$ for $c=c_{*}$. Let $H(\lambda):=F(\lambda)\left(\lambda_{1}-\lambda\right)^{k+1}$, then we have

$$
H(\lambda)=\frac{\left(\lambda^{2}-c_{*} \lambda-1\right) Q_{2}(\lambda)+g^{\prime}(0)\left[e^{-\lambda c \tau}-1\right] Q_{1}(\lambda)}{P(\lambda) /\left(\lambda_{1}-\lambda\right)^{k+1}}-\left(\lambda_{1}-\lambda\right)^{k+1} \int_{0}^{+\infty} \phi_{2}(\xi) e^{-\lambda \xi} d \xi,
$$

since $\phi_{2}(\xi)<\infty$ and $\left(\lambda_{1}-\lambda\right)^{k+1} \int_{0}^{+\infty} \phi_{2}(\xi) e^{-\lambda \xi} d \xi$ is analytic for $\operatorname{Re} \lambda>0$. Note that $\lambda_{1}$ is the smallest positive simple solution of $P(\lambda)=0$ for $c>c^{*}$. Therefore, $\left(\lambda_{1}-\lambda\right)^{k+1} / P(\lambda)$ is analytic for $0<\operatorname{Re} \lambda \leqslant \lambda_{1}$. In addition, we know $\left(\lambda_{1}^{2}-c \lambda_{1}-1\right)<0, Q_{i}\left(\lambda_{1}\right)>0$ and $P(\lambda) /\left(\lambda_{1}-\lambda\right)^{k+1}<0$ at near $\lambda=\lambda_{1}$ for $i=1,2$, it follows that $\mathrm{H}\left(\lambda_{1}\right)>0$.

Notice that $\phi_{2}(\xi)$ may not be monotone increasing on $(-\infty, 0)$, there exists a constant $m>0$ such that $\bar{\phi}_{2}(\xi):=\phi_{2}(\xi) e^{\mathrm{m} \xi}$ is monotone increasing on $(-\infty, 0)$. By $(2.4)$, we have

$$
c \bar{\phi}_{2}^{\prime}(\xi)=\int_{-\infty}^{\xi}\left(m-\frac{\beta}{c}\right) e^{m \xi-\frac{\beta}{c}(\xi-s)} g(\phi(s-c \tau)) d s .
$$

Thus $\bar{\phi}_{2}^{\prime}(\xi)>0$ provided $m>\frac{\beta}{\mathcal{c}_{*}}$. This implies that $\bar{\phi}_{2}(\xi)$ is nondecreasing. It is easy to see that

$$
\overline{\mathrm{F}}(\lambda):=\int_{-\infty}^{0} \bar{\phi}_{2}(\xi) e^{-\lambda \xi} \mathrm{d} \xi=\mathrm{F}(\lambda-\mathrm{m}) .
$$

Using the modified Ikehara's theorem in [1] again, we obtain the existence of $\lim _{\xi \rightarrow-\infty} \frac{\bar{\phi}_{2}(\xi)}{|\xi|^{k} e^{\left(m+\lambda_{1}\right) \xi}}$, where $\mathrm{k}=0$ as $\mathrm{c}>\mathrm{c}_{*}$ and $\mathrm{k}=1$ as $\mathrm{c}=\mathrm{c}_{*}$. Denote

$$
G\left(\lambda_{1}\right):=\lim _{\xi \rightarrow-\infty} \frac{\bar{\phi}_{2}(\xi)}{|\xi|^{k} e^{\left(m+\lambda_{1}\right) \xi}}=\lim _{\xi \rightarrow-\infty} \frac{\phi_{2}(\xi)}{|\xi|^{k} e^{\lambda_{1} \xi}},
$$

and for any $\theta_{2} \in \mathbb{R}$, we obtain

$$
\mathrm{G}\left(\lambda_{1}\right)=\lim _{\xi \rightarrow-\infty} \frac{\phi_{2}\left(\xi+\theta_{2}\right)}{\left|\xi+\theta_{2}\right|^{k} e^{\lambda_{1}\left(\xi+\theta_{2}\right)}}=e^{-\lambda_{1} \theta_{2}} \lim _{\xi \rightarrow-\infty} \frac{\phi_{2}\left(\xi+\theta_{2}\right)}{|\xi|^{k} e^{\lambda_{1} \xi}},
$$

let $\theta_{2}$ is the constant satisfying $e^{\lambda_{1} \theta_{2}} \mathrm{G}\left(\lambda_{1}\right)=1$, then

$$
\lim _{\xi \rightarrow-\infty} \frac{\phi_{2}\left(\xi+\theta_{2}\right)}{|\xi|^{k} e^{\lambda_{1} \xi}}=1,
$$

where $k=0$ as $c>c_{*}$ and $k=1$ as $c=c_{*}$. Similarly, we can obtain that there exists the constant $\theta_{1}$ such that

$$
\lim _{\xi \rightarrow-\infty} \frac{\phi_{2}\left(\xi+\theta_{2}\right)}{|\xi|^{k} e^{\lambda_{1} \xi}}=1
$$

where $\mathrm{k}=0$ as $\mathrm{c}>\mathrm{c}_{*}$ and $\mathrm{k}=1$ as $\mathrm{c}=\mathrm{c}_{*}$. This completes the proof. 


\section{Acknowledgment}

This work was supported by the Youth Fund of Hexi University of China (QN2018008).

\section{References}

[1] J. Carr, A. Chmaj, Uniqueness of travelling waves for nonlocal monostable equations, Proc. Amer. Math. Soc., 132 (2004), 2433-2439. 2

[2] T. Faria, W. Huang, J. Wu, Traveling waves for delayed reaction diffusion equations with nonlocal response, Proc. R. Soc. Lond. Ser. A, 462 (2006), 229-261. 1

[3] T. Faria, S. Trofimchuk, Nonmonotone travelling waves in a single species reaction-diffusion equation with delay, J. Differential Equations, 228 (2006), 357-376. 1

[4] J.-S. Guo, C.-H. Wu, Traveling wave front for a two-component lattice dynamical system arising in competition models, J. Differential Equations, 252 (2012), 4357-4391. 2.2

[5] J. Huang, X. Zou, Existence of traveling wavefronts of delayed reaction diffusion systems without monotonicity, Discrete Contin. Dyn. Syst., 9 (2003), 925-936.

[6] Y. Li, W.-T. Li, Y.-R. Yang, Stability of traveling waves of a diffusive susceptible-infective-removed (SIR) epidemic model, J. Math. Phys., 57 (2016), 28 pages. 1

[7] S. Ma, Traveling wavefronts for delayed reaction-diffusion systems via a fixed point theorem, J. Differential Equations, 171 (2001), 294-314. 1

[8] S. Ma, Traveling waves for nonlocal delayed diffusion equations via auxiliary equations, J. Differential Equations, 237 (2007), 259-277. 1

[9] H. R. Thieme, X.-Q. Zhao, Asymptotic speeds of spread and traveling waves for integral equations and delayed reactiondiffusion models, J. Differential Equations, 195 (2003), 430-470. 1

[10] S.-L. Wu, W.-T. Li, S.-Y. Liu, Oscillatory waves in reaction-diffusion equations with nonlocal delay and crossingmonostability, Nonlinear Anal. Real World Appl., 10 (2009), 3141-3151. 1

[11] S.-L. Wu, S.-Y. Liu, Uniqueness of non-monotone traveling waves for delayed reaction-diffusion equations, Appl. Math. Lett., 22 (2009), 1056-1061. 1

[12] S.-L. Wu, S.-Y. Liu, Traveling waves for delayed non-local diffusion equations with crossing-monostability, Appl. Math. Comput., 217 (2010), 1435-1444. 1

[13] S.-L. Wu, S.-Y. Liu, Existence and uniqueness of traveling waves for non-monotone integral equations with application, J. Math. Anal. Appl., 365 (2010), 729-741. 2

[14] J. Wu, X. Zou, Asymptotic and periodic boundary value problems of mixed FDEs and wave solutions of lattice differential equations, J. Differential Equations, 135 (1997), 315-357.

[15] D. Xu, X.-Q. Zhao, Asymptotic speeds of spread and traveling waves for a nonlocal epidemic model, Discrete Contin. Dyn. Syst. Ser. B, 5 (2005), 1043-1056. 1

[16] X.-Q. Zhao, W. Wang, Fisher waves in an epidemic model, Discrete Contin. Dyn. Syst. Ser. B, 4 (2004), 1117-1128. 1 